\title{
Deposition results from rotating collector diagnostics in JET*
}

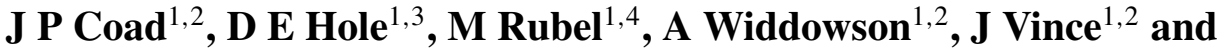 \\ JET-EFDA contributors ${ }^{1}$ \\ ${ }^{1}$ JET-EFDA, Culham Science Centre, Abingdon OX14 3DB, UK \\ ${ }^{2}$ EURATOM/UKAEA Fusion Association, Culham Science Centre, Abingdon OX14 3DB, UK \\ ${ }^{3}$ Department of Engineering and Design, University of Sussex, Brighton BN1 9QH, UK \\ ${ }^{4}$ Royal Institute of Technology, Assoc. EURATOM-VR, 10044 Stockholm, Sweden \\ E-mail: Paul.Coad@ccfe.ac.uk
}

Received 27 October 2009

Accepted for publication 5 November 2009

Published 30 December 2009

Online at stacks.iop.org/PhysScr/T138/014023

\begin{abstract}
Rotating collectors (RC) were installed in JET during the period 2005-2007, each providing a time-resolved deposition pattern on the surface of a rotating silicon disc, which could be analysed once retrieved from the vessel. This paper reports results from the silicon disc removed from the RC located under the load-bearing septum replacement plate in JET in 2007.

Nuclear reaction analysis results of the deposits on the disc have been correlated directly with the pattern of erosion and deposition observed by the quartz microbalance (QMB) located in an equivalent position. The thickest film in the time-resolved region (i.e. deposited in $\sim 60$ pulses) was $\sim 250 \mathrm{~nm}$, and the $\mathrm{Be} / \mathrm{C}$ ratio was generally found to be 0.1 or lower, with two regions where the ratio rose to 0.2 . The deposition observed with the QMB appears to be about a factor of four less.
\end{abstract}

PACS numbers: 52.40.Hf, 52.55.Fa, 82.80.Yc

\section{Introduction}

Post-mortem analysis of divertor tiles gives the most relevant information on erosion/deposition and $\mathrm{H}$-isotope retention in a tokamak, and has been carried out routinely at JET since the first campaign; the results are used inter alia to also study the transport of plasma impurities [1, 2]. However tiles can only be collected during a shutdown, typically once every two years after a campaign containing 5000-7000 pulses with a great variety of discharge types. The analytical information is thus an average over many conditions, and some time-resolved information is also required. The ideal time resolution for measurement of erosion/deposition is every pulse, or, indeed, selected parts of a single pulse. Such resolution is provided in certain locations in JET by quartz micro-balances (QMBs) [3-6]. However, due to requirements on provision of signal and power cables, and restrictions on temperature, these units can operate in JET only in areas of the divertor with connections to electrical feedthroughs and shadowed from direct interaction with the plasma. Owing to

* See the appendix of F Romanelli et al, Fusion Energy Conference 2008 (Proc. 22nd FEC Geneva, 2008) IAEA (2008). the hostile environment in JET, the QMBs are protected from certain types of plasmas by shutters, and are likely to have a finite operating lifetime. This paper describes the use of a simple collector with resolution of approximately one day of operation that can augment the information obtained by other means.

\section{Experimental}

The rotating collector (RC) was developed at JET to provide a simple and more robust time-resolved collector that does not require any electrical connections. The RC diagnostic consists of a collector mounted on a geared mechanism that uses the magnetic field to drive the first gear wheel one step per magnetic pulse by means of a ferromagnetic bar aligning with the field lines. In these first units tested in JET, the gear ratio of the mechanism was such that one geared step was equivalent to a rotation angle of $0.106^{\circ}$. Thus the collector disc rotates $330^{\circ}$ over 3117 pulses, and is exposed through a slit, the width of which determines the time resolution of the collector. The slit (and thus the area exposed on the collector) is a radial strip on the top face, and extending down the side face of 


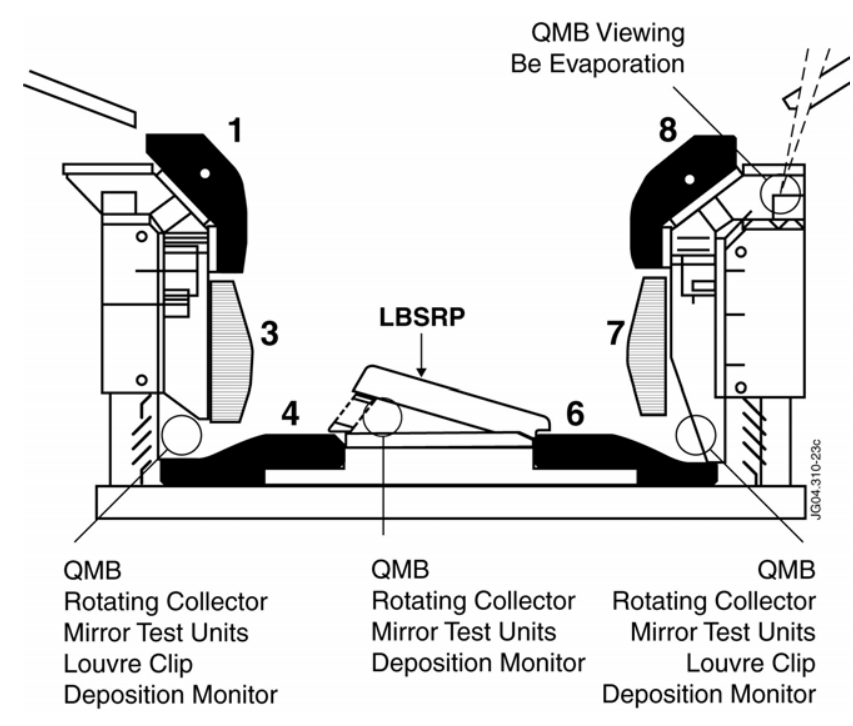

Figure 1. Cross-section of the JET MkII-HD divertor showing the positions of tritium retention diagnostics during the 2005-2007 operational period.

the collector disc. For consistent resolution, the slit should be a radial sector tapering from its full width at the outer circumference to zero on the axis of the collector. However, it is also necessary to consider the spatial resolution of the analysis method that will probe the collector and whether the amount of material collected will be detectable. For the JET RC units the slit width was constant at either 1 or $2 \mathrm{~mm}$; a $1 \mathrm{~mm}$ wide slit results in a resolution at the outer diameter of the collector of $\sim 30$ pulses (typically 1-2 days of JET operation). By altering the gearing ratio and the slit width, collectors can be tailored to suit the planned length of operational exposure and the resolution required.

The collector rotates by one step for every JET pulse with a significant magnetic field at the RC unit. Many JET pulses are commissioning pulses with no, or little, magnetic field, so these have to be discounted when assessing the collector rotation. Although the collectors require no electrical connection, one of the units was fitted with a micro-switch that switched on/off every 10 successful steps $\left(1.06^{\circ}\right)$; this proved useful to provide a quick calibration of collector angle against JET pulse number. Furthermore, by comparing this signal with the toroidal field coil currents it was found that a toroidal field in the range $0.46-0.54 \mathrm{~T}$ was generally sufficient to activate the unit. The lowest toroidal field used in JET experiments is about $0.8 \mathrm{~T}$ and so all normal JET pulses with plasma result in a rotation of the disc.

Five RC units were installed in JET; three in the divertor and two on the main chamber wall at the outer mid-plane. The units in the divertor were positioned at the bottom of the inner and outer divertor carriers and underneath the load-bearing septum replacement plate (LBSRP), as shown in figure 1. The units on the main chamber wall were positioned at the outer mid-plane at sectors $3 \mathrm{E}$ and $4 \mathrm{~B}$. The unit at $3 \mathrm{E}$ was close to a beryllium evaporator head, and the slit was angled towards the evaporator so that a number of evaporations could be individually monitored. Since the collected materials of primary interest are carbon, beryllium and deuterium the collectors were normally cut from crystalline silicon, however the wall unit at $3 \mathrm{E}$ had a carbon collector, in case the rate of beryllium deposition might be substrate dependent. The RC units were exchanged during the 2007 shutdown after exposure for $\sim 7000$ pulses in 2005-2007.

\section{Results}

Results are presented for the unit in the LBSRP. Because of the location of the unit close underneath the load-bearing tile there was no flux to the top surface of the collector through the horizontal part of the slit. The collector was only exposed around its circumferential edge, through the part of the slit (which was $2 \mathrm{~mm}$ wide) oriented vertically, i.e. poloidally, facing the inner divertor wall tile 3. A QMB was mounted alongside the RC; this QMB was unusual in having no shutter (because there was no room for one) so operated continuously, and the data can thus be compared directly with the RC. The viewing angles in the poloidal direction from the $\mathrm{RC}$ and QMB were slightly different. The line of sight for the QMB extended down from the top of tile 3 and included a glancing angle view of tile 4; the view of the top section of tile 3 is vignetted (not visible by all the sensor). The view from the RC was restricted to the central half of tile 3 , with vignetted views (i.e. from part of the collector) of additional sections towards top and bottom of tile 3. It is assumed that material deposited on the QMB or RC has been sputtered from the target tile at or near the plasma strike point and travels directly to the collector.

Figure 2 includes a photograph of a section of the circumference of the RC disc while mounted for analysis. Deposition on the silicon collector was measured by the ion beam analysis method nuclear reaction analysis (NRA) wherein the target is bombarded with a beam of $2.5 \mathrm{MeV}$ helium-3 $\left({ }^{3} \mathrm{He}\right)$ ions and the protons (p) emitted at characteristic energies are analysed. Simultaneous measurements of the amounts of carbon $(\mathrm{C})$, beryllium $(\mathrm{Be})$ and deuterium $\left({ }^{2} \mathrm{H}\right)$ in the outermost microns can then be made via the reactions ${ }^{12} \mathrm{C}\left({ }^{3} \mathrm{He}, \mathrm{p}\right){ }^{14} \mathrm{~N},{ }^{9} \mathrm{Be}\left({ }^{3} \mathrm{He}, \mathrm{p}\right){ }^{11} \mathrm{~B}$ and ${ }^{2} \mathrm{H}\left({ }^{3} \mathrm{He}, \mathrm{p}\right){ }^{4} \mathrm{He}$. The thickest film in the time-resolved region (i.e. deposited in $\sim 60$ pulses) was $\sim 250 \mathrm{~nm}$, and the $\mathrm{Be} / \mathrm{C}$ ratio was generally found to be 0.1 or lower, with two regions where the ratio rose to 0.2 . This compares with the ratio of $\mathrm{Be} / \mathrm{C}$ to be found on tile 3 by post-mortem analysis of $0.5-1.0[1,2]$, and is further evidence that a major mechanism for erosion at tile 3 is chemical sputtering by low energy ions (which preferentially removes carbon). There was a long commissioning period at the start of the 2005-2007 JET campaign due to various operational problems, so unfortunately there was no discernable deposition for the first $\sim 1000$ pulses; this represents one-third of the total useful lifetime of the collector.

Data from the QMB in the LBSRP has been presented previously [5, 6] and is included in figure 2. There was a long period with no change in crystal frequency, corresponding to the extended commissioning mentioned above. However, there were then two periods when the frequency difference between the measuring crystal and a shielded reference crystal increased steadily, with a period between, and a short period afterwards of reducing frequency difference; the former indicate 'integral deposition' and the latter 'integral 


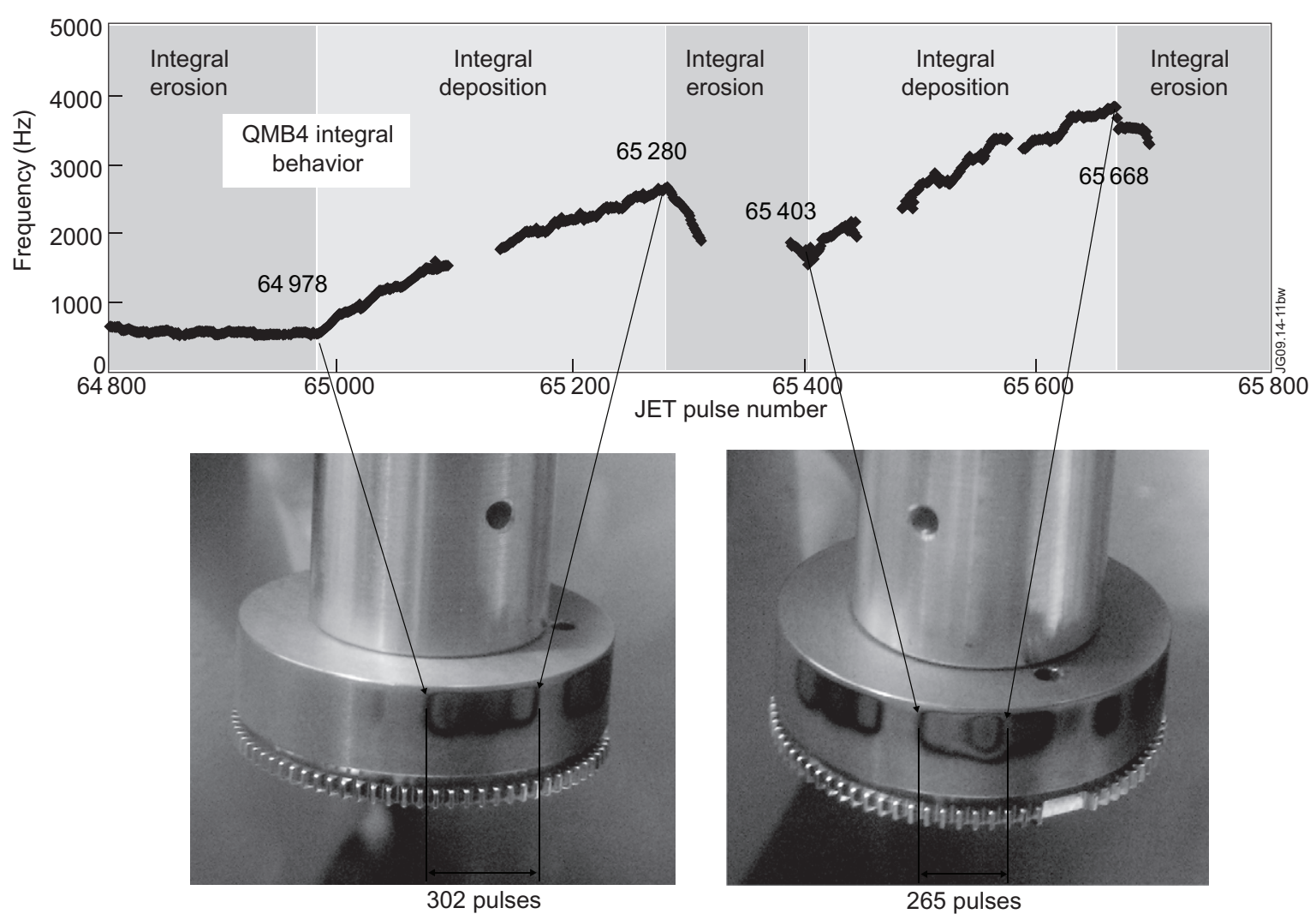

Figure 2. Correlation of a photograph of part of the RC with QMB data from a similar location.

erosion' [6]. Discontinuities in the QMB data are periods when data were not recorded, however, the inherent response frequency of the sensor continues to change during such periods and the updated integrated value will appear automatically when measurements resume. Two of the areas of deposition on the RC correlate with the 'integral deposition' periods on the QMB as shown in figure 2. The carbon and deuterium analyses from NRA around the RC collector are shown at the bottom of figure 3 , together with the same QMB frequency change as shown in figure 2. The change of frequency by $\sim 2000 \mathrm{~Hz}$ in each of the periods of 'integrated deposition' corresponds to $\sim 0.9 \times 10^{18} \mathrm{C}$ atoms cm$^{-2}$ of deposition [6], while the RC data indicate an integrated deposition of $\sim 3.5 \times 10^{18} \mathrm{C}$ atoms $\mathrm{cm}^{-2}$ in the same period. Once the $\mathrm{RC}$ collector has rotated so that a region of deposition is no longer exposed through the slit, it can no longer be eroded by bombarding particles. However, the deposit on the QMB crystal is always exposed, so can be eroded (probably by energetic deuterium ions or neutrals) during discharges when there is little or no flux of impurity particles. This may explain the periods of net erosion seen by the QMB, but cannot explain the difference in deposition rate within the periods of deposition, since some part of the deposit on the collector will have been exposed to the same fluxes as the QMB crystal. The more likely reason for the discrepancy is that the QMB crystal gets very hot during the plasma pulse as it is in poor thermal contact with its surroundings which limits the deposition rate. It also appears that the amounts collected at the inner and outer divertor QMBs are less than might be expected considering the amount of deposition on surrounding surfaces. The QMB in the LBSRP exceeded its operational limit during pulse 65697 , whereas the RC continued to pulse $\sim 66800$.

Figure 3 also shows the strike point positions (as distance $(Z)$ below the JET mid-plane) for the pulses during which the QMB and RC were operational; an outline of the inner divertor tiles to the left of the main plot shows how the $Z$-values relate to tile position. As was noted in [6], the periods of 'integrated deposition' clearly correlate with strike point positions on the lower part of tile 3 , and the periods of 'integrated erosion' with strike point positions on tile 4 . It is unlikely that significant flux will travel to the QMB when the strike point is on tile 4 given the very low angle to the eroding surface required, so erosion at the crystal either thermally or by interaction with deuterium dominates. Note that the RC does not have a line of sight to tile 4 , so that the collector remained clean when the strike point was on tile 4. The continuing $\mathrm{RC}$ data after the demise of the QMB show that the regions of deposit correlate best with strike points on the lower half of tile 3 , rather than strike points anywhere on tile 3 , as may be expected from the viewing angle of the RC. By pulse $\sim 66700$ the collector had completed its rotation, so the large deposition peak centred there represents the integrated flux for the remaining $\sim 4000$ pulses in the 2005-2007 campaign.

\section{Conclusions}

RCs can be used anywhere in the machine since they rely only on the magnetic field to drive the rotation; however, we have found it useful to have a micro-switch to count the number of steps. The RC gives a time-resolved monitor of deposition in the tokamak, and the resolution (in terms of number of pulses in each analysis point) can be varied by adjusting the speed of 


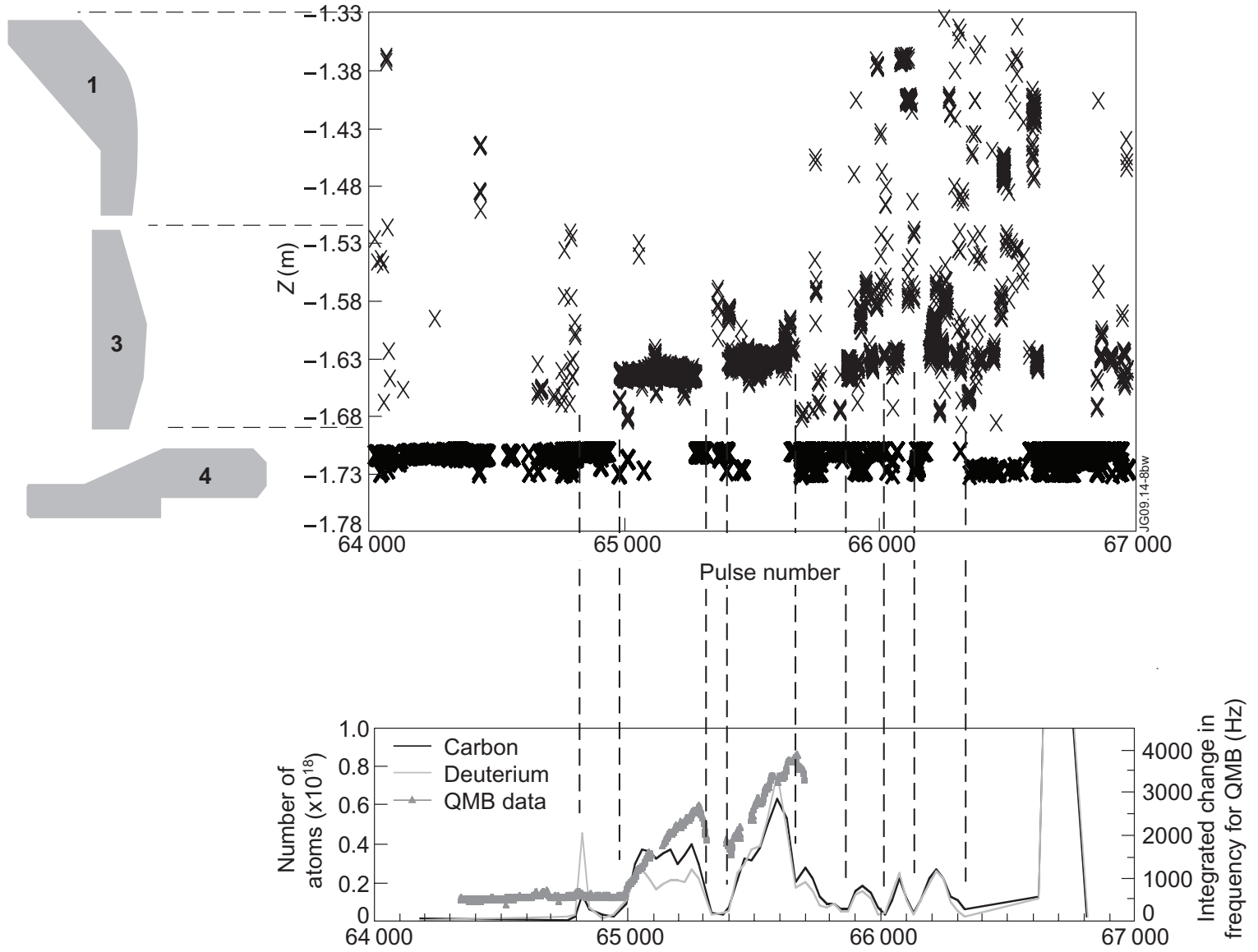

Figure 3. The inner strike point position for JET pulses plotted against vertical distance (referenced to the JET mid-plane). The positions of the inner divertor tiles are indicated to the left. The RC and QMB data show deposition for strike points low on Tile 3-the scale on the left of the lower part of the figure is the number of atoms on the $\mathrm{RC}\left(\times 10^{18}\right)$ and on the right is the change in QMB frequency (in Hz).

rotation and size of exposure slit. For the JET collectors the resolution range was $30-60$ pulses.

The RC located in the LBSRP shows deposition synchronized with net deposition on a QMB located in a similar position. The rate of deposition was greater on the RC than on the QMB crystal. This limited collection efficiency by the QMBs may be due to the high temperature reached by the sensing crystal (which is almost in thermal isolation) during a plasma pulse, a problem that does not exist for the RCs.

\section{Acknowledgments}

This work was funded jointly by the UK Engineering and Physical Sciences Research Council and by the European
Communities under the Contract of Association between EURATOM and UKAEA. The views and opinions expressed herein do not necessarily reflect those of the European Commission.

Published under licence from EURATOM.

\section{References}

[1] Coad J P et al 2006 Nucl. Fusion 46 350-66

[2] Coad J P et al 2007 J. Nucl. Mater. 363-365 287-93

[3] Coad J P et al 2005 Fusion Eng. Des. 74745

[4] Esser H G et al 2007 J. Nucl. Mater. 363-365 146-51

[5] Esser H G et al 2007 34th European Physical Society Conference on Plasma Physics (Warsaw, Poland, July 2007)

[6] Esser H G et al 2009 doi:10.1016/jnucmat.2009.01.153 